Author affiliations appear at the end of this article.

Published at ascopubs.org/journal/jco on October 31, 2016.

Support information appears at the end of this article.

Clinical trial information:

ACTRN12609000683235.

Corresponding author: Janette L. Vardy, MD, Concord Cancer Centre, Concord Repatriation General Hospital, Hospital Rd, Concord, New South Wales, 2139, Australia; e-mail: janette.vardy@sydney. edu.au.

C 2016 by American Society of Clinical Oncology

0732-183X/17/3502w-217w/\$20.00

\title{
Evaluation of a Web-Based Cognitive Rehabilitation Program in Cancer Survivors Reporting Cognitive Symptoms After Chemotherapy
}

Victoria J. Bray, Haryana M. Dhillon, Melanie L. Bell, Michael Kabourakis, Mallorie H. Fiero, Desmond Yip, Frances Boyle, Melanie A. Price, and Janette L. Vardy

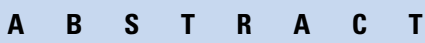

\section{Purpose}

Cognitive impairment is reported frequently by cancer survivors. There are no proven treatments. We evaluated a cognitive rehabilitation program (Insight) and compared it with standard care in cancer survivors self-reporting cognitive symptoms.

\section{Patients and Methods}

We recruited adult cancer survivors with a primary malignancy (excluding central nervous system malignancies) who had completed three or more cycles of adjuvant chemotherapy in the previous 6 to 60 months and reported persistent cognitive symptoms. All participants received a 30-minute telephone consultation and were then randomly assigned to the 15-week, home-based intervention or to standard care. Primary outcome was self-reported cognitive function (Functional Assessment of Cancer Therapy Cognitive Function [FACT-COG] perceived cognitive impairment [PCI] subscale): difference between groups after intervention (T2) and 6 months later (T3).

\section{Results}

A total of 242 participants were randomly assigned: median age, 53 years; $95 \%$ female. The primary outcome of difference in FACT-COG PCl was significant, with less $\mathrm{PCl}$ in the intervention group at T2 $(P<.001)$. This difference was sustained at T3 $(P<.001)$. At T2, there was a significant difference in all FACT-COG subscales, favoring the intervention. Neuropsychological results were not significantly different between the groups at T2 or T3. There were significantly lower levels of anxiety/ depression and fatigue in the intervention group at T2. There were significant improvements in stress in the intervention group at both time points. There was no significant difference in quality of life between the groups at T2, but the intervention group had better quality of life at T3.

\section{Conclusion}

The intervention, Insight, led to improvements in cognitive symptoms compared with standard care. To our knowledge, this is the first large randomized controlled trial showing an improvement in selfreported cognitive function in cancer survivors, indicating that this intervention is a feasible treatment.

\section{J Clin Oncol 35:217-225. (c) 2016 by American Society of Clinical Oncology}

\section{INTRODUCTION}

Up to $70 \%$ of patients with cancer report cognitive symptoms after chemotherapy. ${ }^{1}$ Several studies have shown that self-reported cognitive impairment is associated with increased anxiety, depression, and fatigue and poorer quality of life (QOL). ${ }^{2}$ However, most have found a weak association between cognitive symptoms and objective cognitive impairment on neuropsychological testing. ${ }^{3}$ The cause remains poorly understood, and there are no proven treatments. ${ }^{4}$

Cognitive rehabilitation refers to behaviorally orientated interventions designed to improve performance in cognitive and functional domains, thereby enhancing an individual's functional capacity. These interventions have been shown to improve cognitive function, goal attainment behavior, memory problem solving, and psychosocial functioning in patients with traumatic brain injury. ${ }^{5}$ Retraining programs work best in those with mild cognitive impairment who are sufficiently functional to engage in the program and apply the training to real-world demands. ${ }^{6}$ These characteristics are often seen in cancer survivors.

Evaluation of cognitive rehabilitation programs in patients with cancer has been limited. Ferguson et $\mathrm{al}^{7}$ evaluated a cognitive-behavioral treatment in 
a single-arm pilot study in 29 breast cancer survivors reporting cognitive symptoms 8 years after chemotherapy. Participants had improvements in cognitive symptoms, neuropsychological performance, and QOL after the intervention, which were sustained at 6 months. In a follow-up wait-list control study, the researchers found improved verbal memory and QOL after the intervention. $^{8}$

A wait-list randomized controlled trial (RCT) by Kesler et $\mathrm{al}^{9}$ of an online cognitive training program in 41 patients with breast cancer an average of 6 years after treatment suggested efficacy of cognitive training with improvement in cognitive flexibility, verbal fluency, and processing speed. There were also improvements in self-reported planning, organization, and task monitoring.

The Web-based cognitive rehabilitation program, Insight, and its companion program have demonstrated benefits in populations without cancer and have been pilot tested in patients with cancer. ${ }^{10-12}$ We conducted an RCT evaluating the efficacy of Insight compared with standard care in cancer survivors self-reporting cognitive symptoms after completion of curative chemotherapy for a primary solid malignancy.

\section{PATIENTS AND METHODS}

This pragmatic longitudinal RCT was conducted at 18 Australian sites. Participants were also able to self-refer via two national breast cancer organizations. Institutional ethics approval was obtained at all sites, and written informed consent was obtained from participants.

Eligible participants were $\geq 18$ years old with any solid primary tumor (excluding central nervous system malignancies), who had received definitive treatment of their primary malignancy, including three or more cycles of chemotherapy completed within the previous 6 to 60 months. Participants had to self-report cognitive impairment, indicated by changes in concentration and/or memory, on the two-item European Organization for Research and Treatment of Cancer QLQ-C30 Cognitive Functioning scale. ${ }^{13}$ To meet the eligibility criteria, participants had to rate their cognitive complaints as "quite a bit" or greater in one or both domains. Adjuvant endocrine treatments for patients with breast cancer were permitted, but radiotherapy and targeted therapies had to be completed $\geq 12$ weeks before study entry. Participants required written English fluency to the equivalent of year 8 education. Access to computer and Internet facilities was mandated.

Participants with evidence of local recurrence or metastatic disease or who had had prior malignancy within the previous 5 years (with the exception of nonmelanomatous skin cancer, cervical cancer in situ, and the cancer of interest) were excluded. Those with an unstable psychiatric condition or current major cognitive disorder were excluded. Psychotropic medications were permitted if participants were receiving a stable dose.

Participants were randomly assigned (1:1) to the Insight intervention or to a control group. Random assignment was managed centrally, using an interactive voice response system. Treatment allocation was determined by minimization. Stratification was for primary tumor type, and in patients with breast cancer, hormonal therapy use.

\section{Telephone Consultation}

Before random assignment, all participants participated in a 30-minute structured telephone consultation outlining cognitive compensatory strategies. Standardized scripts were developed outlining cognitive training strategies in four areas: general cognition, memory, concentration, and multitasking. Printed copies of all scripts were mailed to participants.

\section{Intervention Group}

Insight from Posit Science is a computerized neurocognitive learning program that is based on the neuroplasticity model. It uses adaptive exercises targeting processing systems aimed at improving cognition through speed and accuracy of information processing. ${ }^{14}$ It targets cognitive domains including visual precision, divided attention, working memory, field of view, and visual processing speed, which are frequently affected in patients with cancer. ${ }^{14,15}$ The program was provided in compact disc format. Recommended training time was four 40-minute sessions/week for 15 weeks, for a total of 40 hours. The program had a built-in measure of compliance.

\section{Control Group}

Participants in the control group received standard care as per their treating physician.

\section{Assessments}

Assessments were completed at home at baseline (T1), after the 15-week intervention (T2), and 6 months later (T3). Patient-reported outcome (PRO) questionnaires were mailed to participants. Neuropsychological test files were returned via e-mail.

\section{Measures and Evaluations}

The primary outcome was self-reported cognitive function as assessed by the Functional Assessment of Cancer Therapy Cognitive Function version 3 (FACT-COG) questionnaire. The 37-item FACT-COG has acceptable reliability and validity and has been used widely in cancer populations. ${ }^{16}$ It comprises four subscales: perceived cognitive impairments (PCI), perceived cognitive abilities, impact of PCI on QOL, and comments from others on cognitive function. The primary outcome score was the FACT-COG PCI score at T2.

The main secondary outcome was objective neuropsychological function as assessed by Cogstate, an 18-minute computerized battery comprising seven tests evaluating processing speed, decision making, working memory, executive function, continuous performance, matching, and new learning. ${ }^{17}$ Cogstate was selected because it evaluates multiple domains and can be completed independently at home. It has been validated against extensive neuropsychological tests in longitudinal studies, with good reliability. ${ }^{18}$ A total score was derived by standardizing each of the $z$ scores with a mean of 0 and a standard deviation of 1 .

Other secondary outcomes included anxiety/depression (12-item General Health Questionnaire), QOL (FACT-General), fatigue (FACTFatigue subscale), and stress (14-item Perceived Stress Scale).

All questionnaires have been validated in cancer populations. ${ }^{19-22}$

\section{Statistical Analysis}

Linear mixed models with fixed effects of baseline outcome measurement (analysis of covariance), time (categorical), intervention arm, time, arm $\times$ time, and stratification and a random effect of participant were used to model each continuous outcome. ${ }^{23}$ Tests of treatment effect were undertaken within these models using contrasts, which estimated the difference between the groups at each postbaseline time point, with CIs. The original stratification was based on primary tumor type, and hormonal therapy use in patients with breast cancer, resulting in five strata. Because of limited numbers in some strata, we reduced the strata to three: breast cancer with hormone therapy, breast cancer without hormone therapy, and other cancers. To investigate the sensitivity of results to missing data, we (1) performed multiple imputation for the primary outcome, and (2) fit an adjusted model using baseline variables found to be associated with missing data or the primary outcome.

Statistical significance was set at .05 for the primary outcome of PCI. All other outcomes were considered significant at .01, to informally account for multiple comparisons. Analyses were performed in SAS version 9.4 (SAS Institute, Cary, NC). 
Sample size calculations were based on the comparison of the primary outcome, FACT-COG PCI, between the two groups, at T2. Because there was no available minimal important difference (MID) for this scale, we estimated the value of 6.5 on the basis of the FACT-General MID findings of Webster et al. ${ }^{24}$ We assumed a standard deviation of 13, a twosided $t$ test, a type I error of $0.05,90 \%$ power, and equal group sizes. This is just under $10 \%$ of the range, a commonly used rule of thumb for MIDs. Assuming 30\% attrition, the final sample size was 216 .

\section{RESULTS}

Between November 2009 and March 2014, 386 participants were assessed for study eligibility. Of these, 242 participants were randomly assigned: 121 to the intervention, Insight, and 121 to the control group (Fig 1). At T2, primary outcome data were available for 192 (79\%): 94 in the intervention group (78\%) and 98 control subjects $(81 \%)$. At T3, primary outcome data were available for 184 (76\%): 95 in the intervention (79\%) and 89 control subjects (74\%). These rates were not statistically different $(P=.87)$.

Baseline characteristics were well balanced between the groups (Table 1). Median age was 53 years (23 to 74 years); 230 (95\%) were female; $216(89 \%)$ had breast cancer; and $13(5 \%)$ had colorectal cancer. The mean time since completion of chemotherapy was 27 months (6 to 60 months).

At T2, there were statistically significant differences in all FACT-COG subscales in the intervention group compared with the control group (Fig 2). The primary outcome of difference between the groups on the FACT-COG PCI was statistically significant, with less PCI in the intervention group at T2 $(-7.47 ; 95 \% \mathrm{CI},-10.80$ to $-4.13 ; P<.001)$. This difference was sustained at T3 $(-6.48$; $95 \%$ CI -9.85 to $-3.11 ; P=.001)$. Perceived cognitive abilities were significantly better in the intervention group at T2 (3.34; $95 \%$ CI, 1.98 to $4.70 ; P<.001)$ and T3 $(2.88 ; 95 \%$ CI, 1.50 to 4.25 ; $P<.001)$. Participants in the intervention group reported less impact on their QOL from PCI at T2 $(-1.20 ; 95 \% \mathrm{CI},-2.20$ to $-0.20 ; P=.02)$ and T3 $(-1.0 ; 95 \% \mathrm{CI},-2.10$ to $0.01 ; P=.06)$. Participants in the intervention group reported fewer comments from others suggesting that they had cognitive impairment at T2 $(-0.71 ; 95 \% \mathrm{CI},-1.40$ to $-0.02 ; P=.04)$, but there were no differences between the groups at T3 $(-0.38 ; 95 \% \mathrm{CI},-1.08$ to $0.33 ; P=.29$ ).

The major secondary outcome was neuropsychological function, and at T2 and T3, there were no significant differences between the groups in total score or in the six cognitive domains

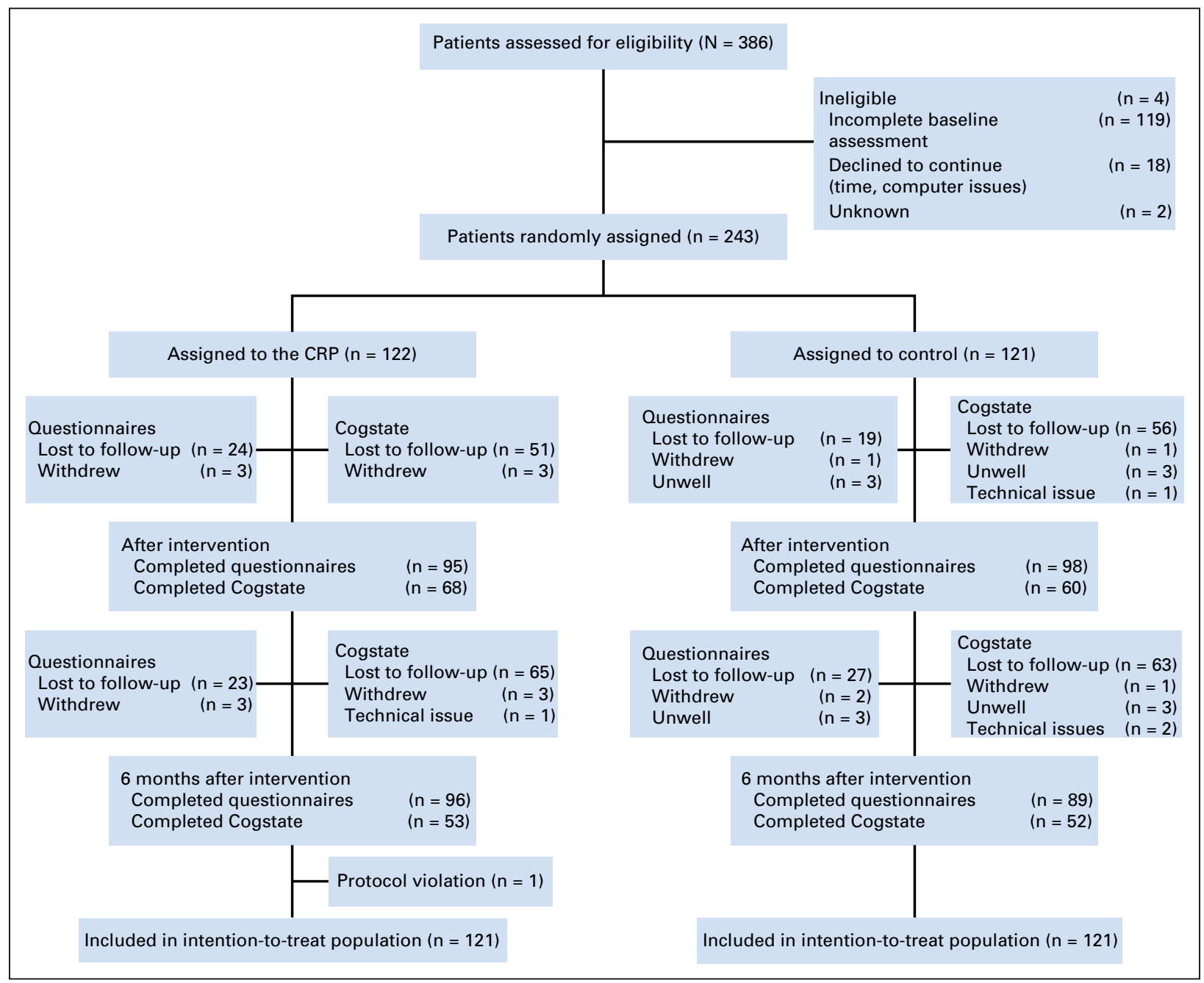

Fig 1. CONSORT diagram. CRP, cognitive rehabilitation program. 


\begin{tabular}{|c|c|c|}
\hline Characteristic & $\operatorname{CRP}(n=121)$, No. $(\%)$ & Control $(n=121)$, No. $(\%)$ \\
\hline \multicolumn{3}{|l|}{ Sex } \\
\hline Female & $116(96)$ & $114(94)$ \\
\hline Male & $5(4)$ & $7(6)$ \\
\hline Age, years, median (range) & $52(23-74)$ & $54(31-74)$ \\
\hline \multicolumn{3}{|l|}{ Married/de facto relationship } \\
\hline Yes & 95 (79) & $97(80)$ \\
\hline No & $17(14)$ & $16(13)$ \\
\hline Unknown & $9(7)$ & $8(7)$ \\
\hline Education, years, median (range) & $14(8-19)$ & $12(3-19)$ \\
\hline \multicolumn{3}{|l|}{ English as first language } \\
\hline Yes & $117(97)$ & $117(97)$ \\
\hline No & $4(3)$ & $4(3)$ \\
\hline \multicolumn{3}{|l|}{ Smoking history } \\
\hline Never & $72(60)$ & $63(52)$ \\
\hline Previous & 47 (39) & $51(42)$ \\
\hline Current & $2(2)$ & $6(5)$ \\
\hline Unknown & $0(0)$ & $1(1)$ \\
\hline \multicolumn{3}{|l|}{ Primary tumor type } \\
\hline Breast & $108(89)$ & $108(89)$ \\
\hline Colorectal & $6(5)$ & $7(6)$ \\
\hline Gynecologic & $2(2)$ & $3(2)$ \\
\hline Lymphoma & $2(2)$ & $1(1)$ \\
\hline Thoracic & $2(2)$ & $1(1)$ \\
\hline Upper GI & $0(0)$ & $1(1)$ \\
\hline Other & $1(1)$ & $0(0)$ \\
\hline Time since completion of chemotherapy, months, mean (range) & $27(6-57)$ & $27(6-60)$ \\
\hline \multicolumn{3}{|l|}{ Radiotherapy } \\
\hline Yes & $86(71)$ & $78(64)$ \\
\hline No & $35(29)$ & $43(36)$ \\
\hline \multicolumn{3}{|l|}{ Immune therapy } \\
\hline Yes & $30(25)$ & $24(20)$ \\
\hline No & $91(75)$ & $97(80)$ \\
\hline \multicolumn{3}{|l|}{ Hormone therapy } \\
\hline Tamoxifen & $34(28)$ & $36(30)$ \\
\hline Letrozole & $15(12)$ & $18(15)$ \\
\hline Anastrazole & $29(24)$ & $25(21)$ \\
\hline Other & $6(5)$ & $6(5)$ \\
\hline None & $37(31)$ & $36(30)$ \\
\hline \multicolumn{3}{|l|}{ Previous neurologic history* } \\
\hline Yes & $20(17)$ & $30(25)$ \\
\hline No & $101(83)$ & $91(75)$ \\
\hline \multicolumn{3}{|l|}{ Ever used antidepressants } \\
\hline Yes & $54(45)$ & $54(45)$ \\
\hline No & $67(55)$ & $67(55)$ \\
\hline \multicolumn{3}{|l|}{ Current use of antidepressants } \\
\hline Yes & $28(23)$ & $25(21)$ \\
\hline No & $93(77)$ & $96(79)$ \\
\hline \multicolumn{3}{|l|}{ Patient-reported outcomes, mean (SD) } \\
\hline Perceived cognitive impairments (FACT-COG PCI) & $38.6(14.3)$ & $41.9(15.1)$ \\
\hline Perceived cognitive abilities (FACT-COG PCA) & $12.0(5.0)$ & $12.5(5.6)$ \\
\hline Comments from others (FACT-COG comments) & $3.0(3.6)$ & $3.3(3.7)$ \\
\hline Cognitive quality of life (FACT-COG QOL) & $7.5(4.2)$ & $7.7(4.4)$ \\
\hline Fatigue (FACT-F) & $31.4(11.5)$ & $32.9(10.9)$ \\
\hline Quality of life (FACT-G) & 76.5 (15.3) & $77.1(14.1)$ \\
\hline Anxiety and depression (GHQ) & $26.9(5.8)$ & $26.9(6.0)$ \\
\hline Stress (PSS) & $25.2(5.0)$ & $24.5(4.8)$ \\
\hline \multicolumn{3}{|c|}{$\begin{array}{l}\text { Abbreviations: CRP, cognitive rehabilitation program; FACT-COG, Functional Assessment of Cancer Therapy Cognitive Function; FACT-F, Functional Assessment o } \\
\text { Cancer Therapy-Fatigue; FACT-G, Functional Assessment of Cancer Therapy-General; GHQ, General Health Questionnaire; PCA, perceived cognitive abilities; PCI } \\
\text { perceived cognitive impairments; PSS, Perceived Stress Scale; QOL, quality of life; SD, standard deviation. } \\
\text { *Defined as held back a grade in school; required remedial help at school; diagnosed with a learning disability; head injury with loss of consciousness with residua } \\
\text { sequelae; history of seizures, dementia, coma, epilepsy, cardiac arrest requiring cardiopulmonary resuscitation; stroke; history of other neurologic risk; history o } \\
\text { significant alcohol abuse. }\end{array}$} \\
\hline
\end{tabular}

(Table 2). This finding should be interpreted with caution because of missing data rates at both time points. On review of PRO data, we found significant benefits favoring the intervention at $\mathrm{T} 2$ in all secondary outcomes, with the exception of QOL (Fig 3). There were lower levels of anxiety/depression in the intervention group at T2 $(-1.78 ; 95 \% \mathrm{CI},-3.29$ to $-0.27 ; P=.02)$, with no significant difference at T3 $(-1.50 ; 95 \% \mathrm{CI},-3.04$ to $0.04 ; P=.06)$. Similarly, there were lower levels of fatigue in the intervention group at T2 


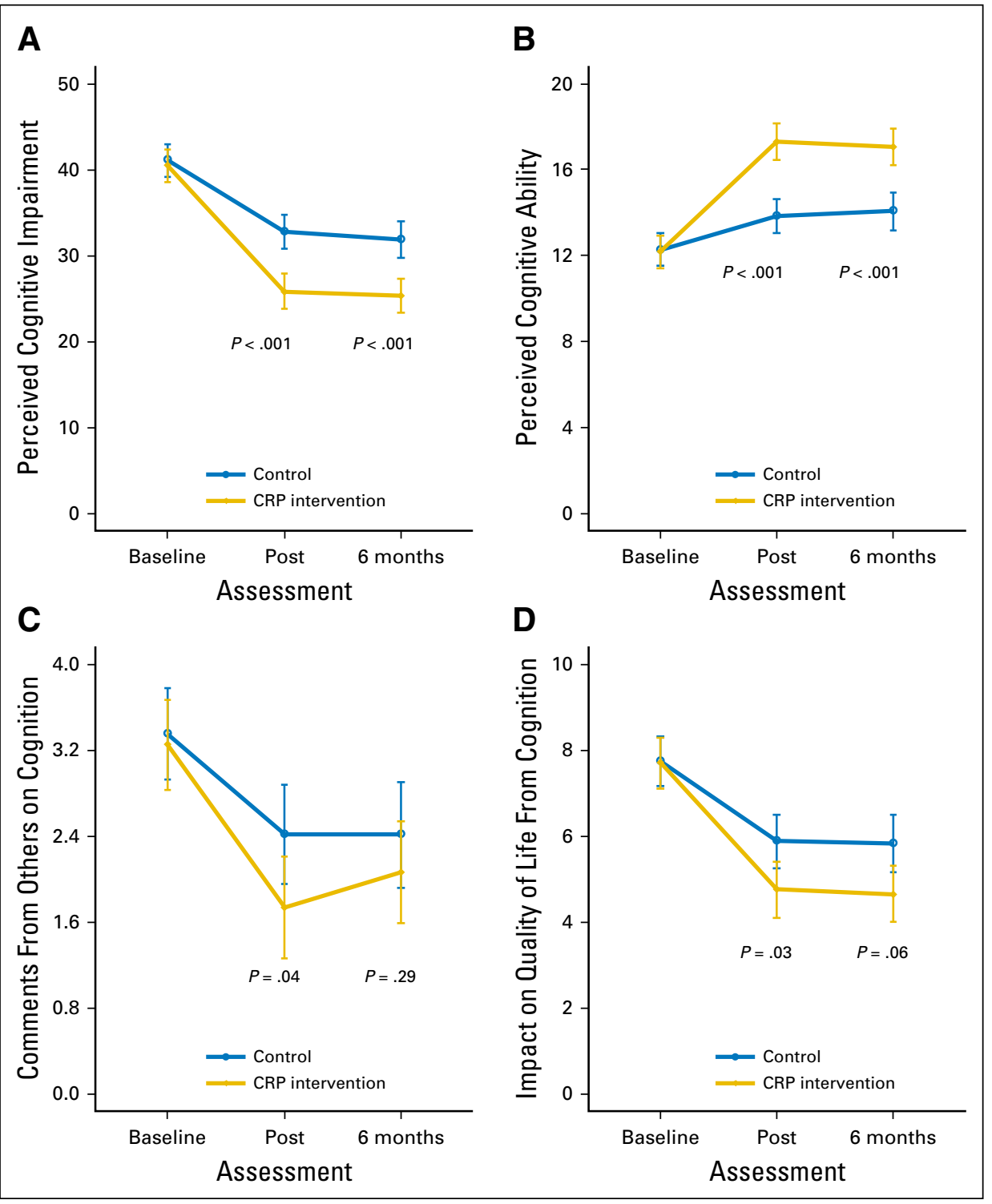

Fig 2. Mean scores for self-reported cognitive function over time, by group. CRP, cognitive rehabilitation program.
(2.44; $95 \%$ CI, 0.25 to $4.62 ; P=.03)$, with no significant difference at T3 $(2.16 ; 95 \% \mathrm{CI},-0.05$ to $4.37 ; P=.06)$. There was no difference in global QOL between the groups at T2 (1.66;95\% $\mathrm{CI},-0.93$ to $4.24 ; P=.21)$, but the intervention group had better global QOL at T3 $(3.39 ; 95 \% \mathrm{CI}, 0.77$ to $6.01 ; P=.01)$. There were benefits in perceived stress in the intervention group at T2 $(-1.30$; $95 \% \mathrm{CI},-2.48$ to $-0.12 ; P=.03)$ and T3 $(-1.85 ; 95 \% \mathrm{CI},-3.05$ to $-0.65 ; P=.01)$. Figure 4 illustrates the $\mathrm{PRO}$ as standardized effect sizes with $95 \%$ CIs. It confirms a clear direction of benefit for the intervention at T2 and T3, with T2 standardized effect sizes of 0.28 for FACT-COG PCI and 0.31 for perceived cognitive abilities.

The sensitivity analysis results (Appendix, online only) were similar to the primary analysis, in both the magnitude of the estimates and the statistical significance, indicating that data were likely to not be informatively missing. Participants who did not complete the T2 assessment had higher rates of antidepressant use and were younger.
Of those randomly assigned to the intervention, 104 (86\%) used the program, and $14 \%$ never started it. The average total training time was 25.08 hours ( 0.19 to 55.82 hours) of a recommended 40 hours. Only 33 participants (27\%) completed the program in the recommended 15-week timeframe. Using regression models, we performed exploratory dose-response analyses for intervention training time with both change in FACT-COG PCI and Cogstate total score. There was no evidence of dose-response for either outcome. Further exploratory analyses compared the FACT-COG PCI change from baseline to T2 for those above and below the mean training time (25.08 hours). Those who trained for $>25.08$ hours had a change of 16.3 points, compared with 12.6 points for those who trained for $<25.08$ hours (difference, 3.7; $95 \% \mathrm{CI},-1.9$ to 9.2 ). We used regression models to explore the factors associated with training time and found that higher baseline anxiety/depression was marginally associated with less training time $(P=.03)$. There was no evidence of association for other baseline variables including fatigue, PCI, and Cogstate score. 


\begin{tabular}{|c|c|c|c|c|c|c|c|}
\hline Cognitive Domain Assessed & Task Name & $\begin{array}{l}\text { Primary Outcome } \\
\text { Measure }\end{array}$ & $\begin{array}{l}\text { Timing of } \\
\text { Assessment }\end{array}$ & $\begin{array}{c}\text { CRP } \\
(n=121)\end{array}$ & $\begin{array}{l}\text { Control } \\
(n=121)\end{array}$ & $\begin{array}{l}\text { Difference } \\
(95 \% \mathrm{Cl})\end{array}$ & $P$ \\
\hline \multirow[t]{3}{*}{ Visual episodic memory } & \multirow{3}{*}{$\begin{array}{l}\text { Continuous paired associate } \\
\text { learning task }\end{array}$} & \multirow[t]{3}{*}{ Total errors* } & T1 & 28.04 & 27.04 & - & - \\
\hline & & & T2 & 33.80 & 28.23 & $5.56(-1.26$ to 12.38$)$ & .11 \\
\hline & & & T3 & 25.87 & 27.09 & $-1.22(-8.88$ to 6.45$)$ & .76 \\
\hline \multirow[t]{3}{*}{ Problem solving and reasoning } & \multirow[t]{3}{*}{ Groton maze learning task } & \multirow[t]{3}{*}{ Total errorst } & T1 & 46.06 & 45.82 & - & - \\
\hline & & & $\mathrm{T} 2$ & 43.47 & 44.51 & $-1.04(-5.03$ to 2.96$)$ & 61 \\
\hline & & & T3 & 41.95 & 45.30 & $-3.36(-7.86$ to 1.15$)$ & .14 \\
\hline \multirow[t]{3}{*}{ Visual learning and memory } & \multirow[t]{3}{*}{ One card learning } & \multirow{3}{*}{$\begin{array}{l}\text { Arcsine proportion } \\
\text { correct }\end{array}$} & T1 & 1.00 & 1.03 & - & - \\
\hline & & & $\mathrm{T} 2$ & 1.05 & 1.00 & $0.05(-0.01$ to 0.11$)$ & .08 \\
\hline & & & T3 & 1.05 & 0.98 & $0.06(-0.00$ to 0.13$)$ & .06 \\
\hline \multirow[t]{3}{*}{ Working memory consolidation } & \multirow[t]{3}{*}{ One back task } & \multirow{3}{*}{$\begin{array}{l}\text { Speed }\left(\log _{10}\right. \\
\text { milliseconds)§ }\end{array}$} & $\mathrm{T} 1$ & 2.89 & 2.90 & - & - \\
\hline & & & $\mathrm{T} 2$ & 2.89 & 2.90 & $-0.00(-0.03$ to 0.02$)$ & .71 \\
\hline & & & T3 & 2.87 & 2.90 & $-0.03(-0.05$ to -0.00$)$ & .05 \\
\hline \multirow[t]{3}{*}{ Working memory consolidation } & \multirow[t]{3}{*}{ Two back task } & \multirow{3}{*}{$\begin{array}{l}\text { Speed }\left(\log _{10}\right. \\
\text { milliseconds)§ }\end{array}$} & T1 & 2.95 & 2.96 & - & - \\
\hline & & & $\mathrm{T} 2$ & 2.98 & 2.97 & $0.01(-0.02$ to 0.04$)$ & .64 \\
\hline & & & T3 & 2.97 & 2.96 & $0.01(-0.02$ to 0.04$)$ & .48 \\
\hline \multirow{3}{*}{$\begin{array}{l}\text { Information processing/psychomotor } \\
\text { function }\end{array}$} & \multirow[t]{3}{*}{ Detection task } & \multirow{3}{*}{$\begin{array}{l}\text { Speed }\left(\log _{10}\right. \\
\text { milliseconds)§ }\end{array}$} & $\mathrm{T} 1$ & 2.56 & 2.57 & - & - \\
\hline & & & $\mathrm{T} 2$ & 2.55 & 2.57 & $-0.02(-0.05$ to 0.02$)$ & .33 \\
\hline & & & T3 & 2.56 & 2.58 & $-0.02(-0.06$ to 0.02$)$ & .27 \\
\hline \multirow[t]{3}{*}{ Attention } & \multirow[t]{3}{*}{ Identification task } & \multirow{3}{*}{$\begin{array}{l}\text { Speed }\left(\log _{10}\right. \\
\text { milliseconds)§ }\end{array}$} & T1 & 2.73 & 2.74 & - & - \\
\hline & & & $\mathrm{T} 2$ & 2.73 & 2.72 & $0.00(-0.01$ to 0.02$)$ & .60 \\
\hline & & & T3 & 2.72 & 2.74 & $-0.02(-0.04$ to -0.00$)$ & .04 \\
\hline \multirow[t]{3}{*}{ Total score } & & & T1 & -0.03 & 0.05 & - & - \\
\hline & & & $\mathrm{T} 2$ & 0.09 & 0.03 & $0.00(-0.07$ to 0.07$)$ & .21 \\
\hline & & & T3 & 0.00 & 0.06 & $-0.02(-0.06$ to -0.22$)$ & .09 \\
\hline
\end{tabular}

Abbreviations: CRP, cognitive rehabilitation program; T1, baseline; T2; after intervention; T3, 6 months after intervention.

* Number of errors made in correctly placing each of the four patterns in their location four times (lower score $=$ better performance).

tTotal number of errors made in attempting to learn the same hidden pathway on five consecutive trials at a single session (lower score = better performance).

¥Accuracy of performance. Arcsine transformation of the square root of the proportion of correct responses (higher score $=$ better performance).

$\S$ Speed of performance. Mean of the $\log _{10}$ transformed reaction times for correct responses (lower score $=$ better performance).

\section{DISCUSSION}

This was a large, longitudinal, RCT evaluating a Web-based cognitive rehabilitation program in patients with cancer reporting cognitive symptoms after chemotherapy. Those randomly assigned to the intervention had improved perceived cognitive function after the intervention that was sustained at 6 months. There were no major differences in objective neuropsychological test results between the groups. Importantly, symptoms of anxiety/ depression, fatigue, and stress were lower in the intervention group on completion of the program, and QOL was improved at 6 months.

To our knowledge, this is the largest RCT to evaluate a cognitive rehabilitation program in patients with cancer. There have been a number of small wait-list control trials of cognitive interventions, predominantly in patients with breast cancer. ${ }^{8,9,25-27}$ These studies have been heterogeneous, with differing primary outcomes and diverse cognitive training strategies.

Von $\mathrm{Ah}$ et $\mathrm{al}^{25}$ conducted a three-arm, single-blind RCT of training in memory and processing speed in 82 breast cancer survivors 6 years after treatment. The groups included group sessions of memory training, the Insight program, and a wait-list group. They found that training of memory and processing speed improved objective cognitive performance. There were also significant improvements in perceived cognitive function, mood, anxiety, fatigue, and QOL in the cognitive training groups compared with the wait-list control group.
Cherrier et $\mathrm{al}^{28}$ conducted an RCT of a 7 -week cognitive rehabilitation intervention in 28 participants with various tumor types, a median of 3 years after treatment. The intervention comprised group sessions including content on memory aids, development of memory skills, and mindfulness meditation. The intervention group had improvements in PCI and ability, less impact of their cognitive symptoms on QOL, and improvements on objective measures of attention.

The major strengths of our study are the RCT design, the large sample size, and assessment of both self-reported and neuropsychological function, both of which are important to cancer survivors and are valid end points. The pragmatic design, with broad eligibility criteria incorporating both sexes, no upper age limit, and patients with any primary solid tumor type, is a strength. The rationale was to ensure that the results were generalizable to the majority of cancer survivors as recommended by the International Cognition and Cancer Task Force. ${ }^{29,30}$ The selection of a home-based intervention and remote assessments was to ensure equity of access to cancer survivors throughout Australia, irrespective of geographical location.

Despite our broad selection criteria, the majority of study participants were patients with breast cancer; this was driven by patient interest and recruitment strategies. The number of participants with other primary tumor types was too small for subgroup analysis. However, there is no inherent mechanism suggesting that this intervention would not yield similar results for other tumor types.

This study has a number of limitations. We acknowledge that the ideal study design would have included a third group randomly 


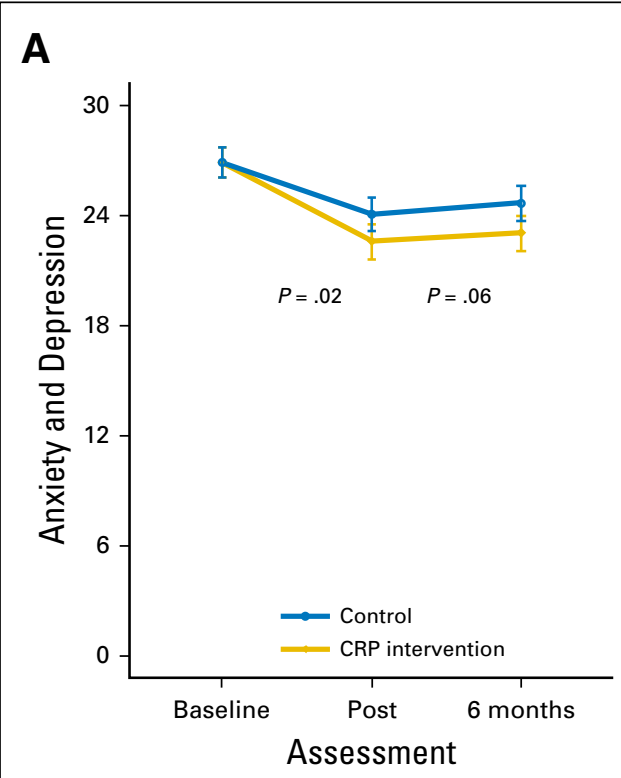

C

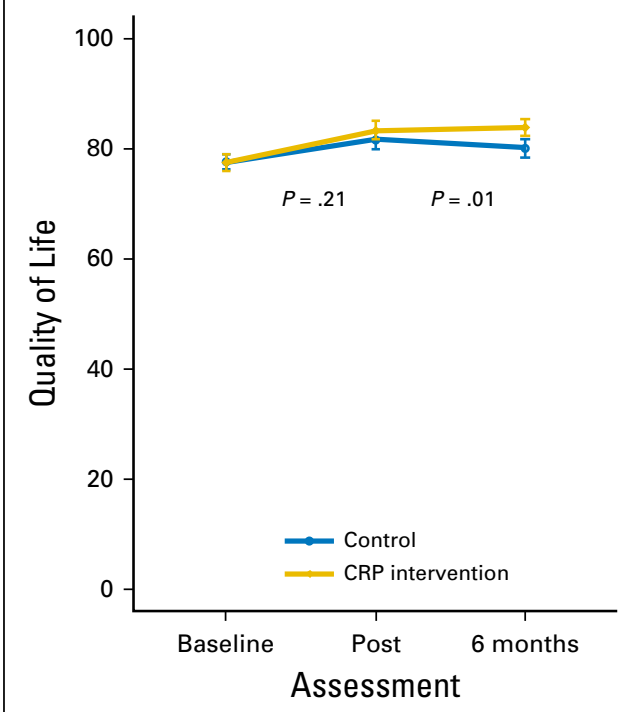

B

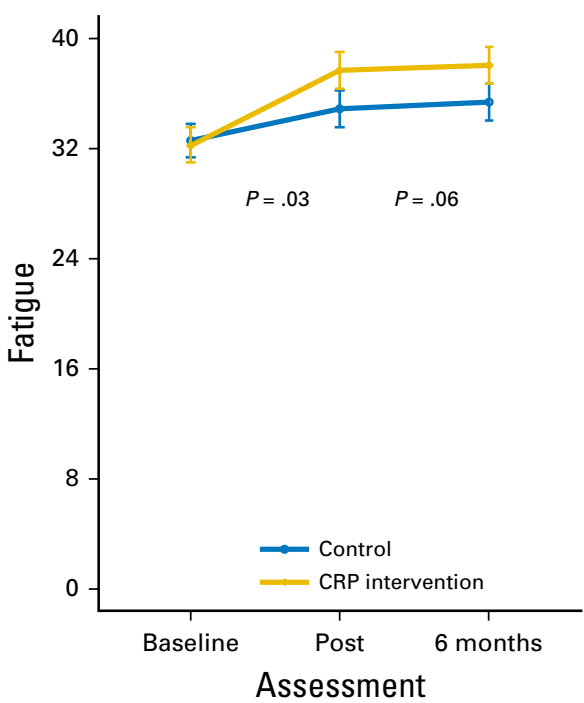

D

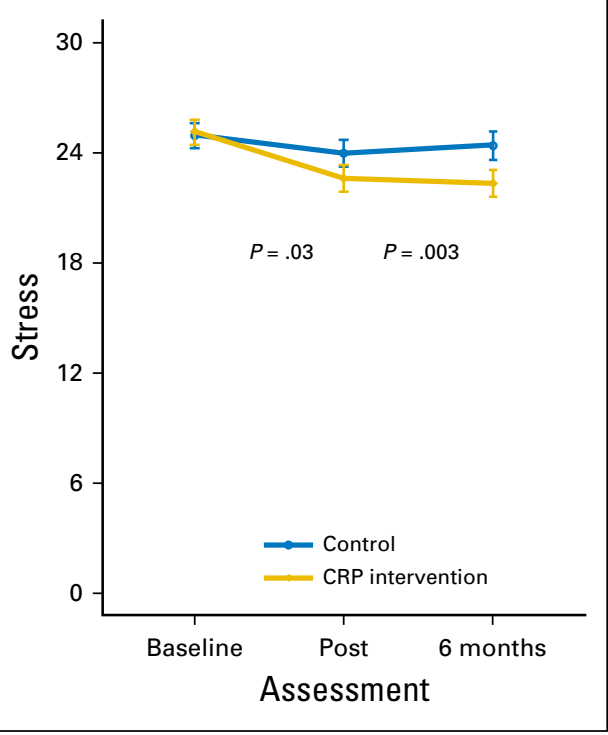

Fig 3. Mean scores for patient-reported outcomes over time, by group. CRP, cognitive rehabilitation program. assigned to a 40-hour nontherapeutic intervention. This was not feasible because of difficulties in ensuring that the intervention would be nontherapeutic, home based, and acceptable to participants and for budgetary and resource considerations. It is possible that simply performing an intervention, or expectation of benefit, may improve self-reported cognitive impairment, but any placebo effect derived on completion of the intervention is likely to diminish over time and to not be sustained at T3. We attempted to control for placebo effect with the telephone consultations with both groups. Although all participants took part in the telephone consultations, differences were seen favoring the intervention group, suggesting a benefit from the program, beyond that of the telephone consultation alone.

Another limitation of the study was inherent in the pragmatic study design. Participants were unsupervised when performing the intervention, and assessments were completed remotely. This had a significant impact on completion rates, particularly for the neuropsychological assessment with Cogstate. This was challenging for several participants because of the required level of computer literacy to install and later reaccess Cogstate. Similarly, some participants experienced technical difficulties using Insight, and with no in-person information technology support available, in some instances these issues were unable to be rectified. This had implications for the use of Insight and the median training time. Reassuringly, the study met its primary end point, with an average training time of 25 hours, rather than the recommended 40-hour training period. With Insight moving to an online platform, this is less likely to be problematic in the future and will enhance translation into practice. Taken together, these issues resulted in missing data. We performed additional statistical analyses to investigate this further (Appendix). 


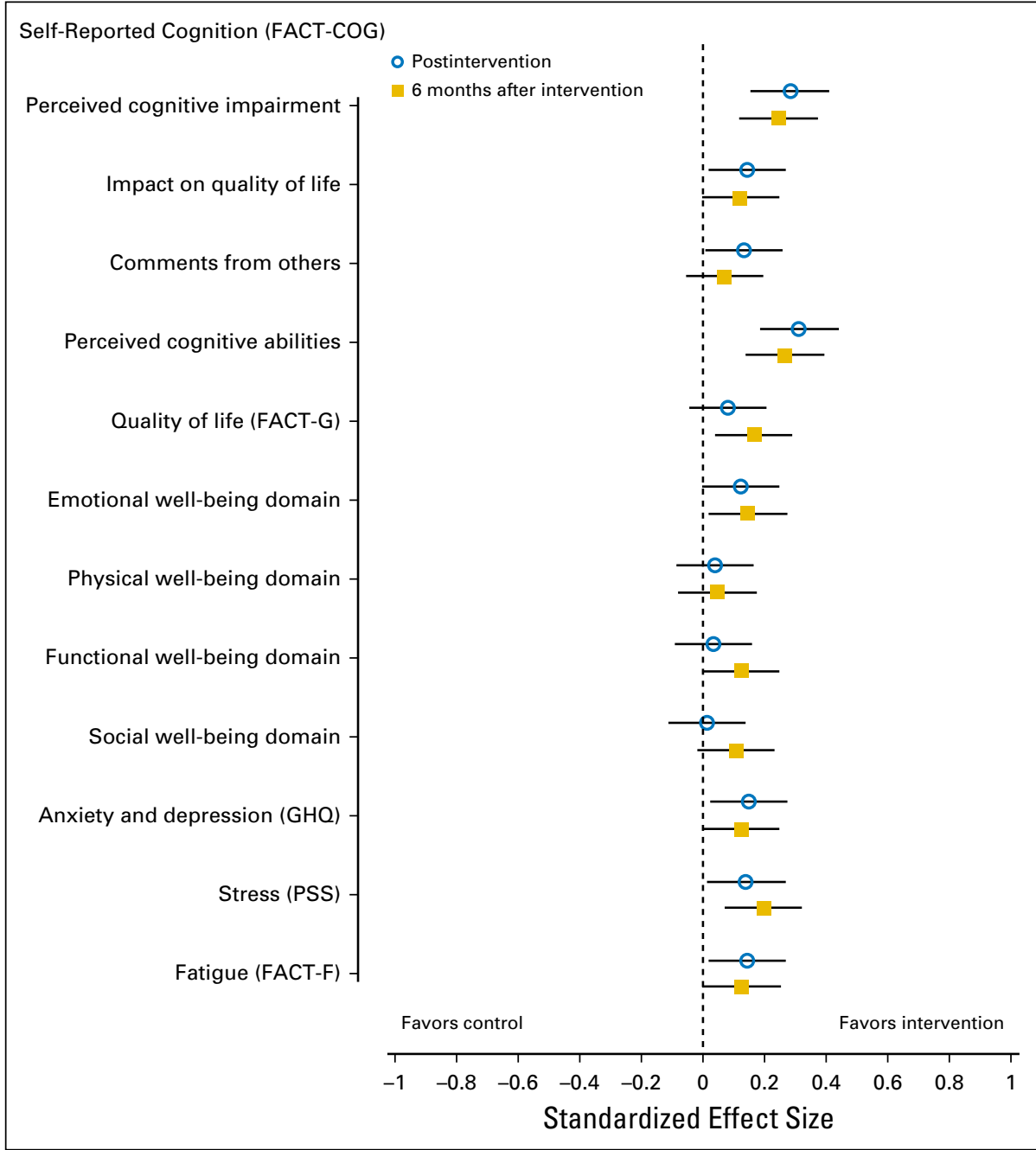

Fig 4. Standardized effect sizes (and $95 \% \mathrm{Cls}$ ) of the difference between treatment groups in patientreported outcomes. Standardized effect sizes $<0.2$ are not likely to be clinically important. FACT-COG, Functional Assessment of Cancer Therapy Cognitive Function; FACT-F, Functional Assessment of Cancer Therapy-Fatigue; FACT-G, Functional Assessment of Cancer Therapy-General; GHQ, General Health Questionnaire; PSS, Perceived Stress Scale.
We found no evidence of a difference in objective neuropsychological results between the groups. One could question whether a difference would have been seen with higher completion rates for neuropsychological testing and if participants had completed the recommended 40-hour training period. However, previous studies have shown a weak association between cognitive symptoms and objective cognitive function. It is possible that different constructs were measured, that the Cogstate battery did not have the sensitivity required to detect the subtle cognitive impairment that cancer survivors typically experience, or that the ideal conditions under which formal neuropsychological testing is performed may not reproduce a cancer survivor's cognitive symptoms.

In line with the findings of previous studies, our results show that self-reported cognitive impairment is associated with increased anxiety/depression and fatigue and poorer QOL. However, the relationship is complex. We do not believe that cognitive symptoms can be explained fully by affective symptoms, because we found sustained improvements in cognitive symptoms in the intervention group at 6 months. We acknowledge that some of the improvements in PRO in the intervention group may have been a result of participants' self-efficacy or an expectancy effect engendered by performing an activity to address their symptoms.

The study collected data on a number of PRO, with multiple tests undertaken at different time points. We acknowledge that some of the trends seen, particularly in the individual cognitive domains of Cogstate and in QOL domains, may be chance findings.

To date, there has been a large unmet need for effective treatment options for cancer survivors experiencing cognitive symptoms after chemotherapy treatment. Previous research has shown cognitive rehabilitation strategies to be feasible, with preliminary evidence of efficacy. Our large RCT adds weight to this evidence, confirming that the use of Insight led to an improvement in cognitive symptoms. Importantly, there were also improvements in $\mathrm{PRO}$, including QOL and reduction in stress, fatigue, and anxiety/depression. The program has the additional advantages of being relatively inexpensive and home based, allowing individuals to direct their own treatment. The program has the potential to provide a new treatment option for patients with cancer with cognitive symptoms, where previously none existed. 


\section{AUTHORS' DISCLOSURES OF POTENTIAL CONFLICTS} OF INTEREST

Disclosures provided by the authors are available with this article at ascopubs.org/journal/jco.

\section{AUTHOR CONTRIBUTIONS}

Conception and design: Victoria J. Bray, Haryana M. Dhillon, Melanie A. Price, Janette L. Vardy
Administrative support: Michael Kabourakis

Provision of study materials or patients: Victoria J. Bray, Haryana M. Dhillon, Desmond Yip, Frances Boyle, Melanie A. Price, Janette L. Vardy Collection and assembly of data: Victoria J. Bray, Haryana M. Dhillon, Michael Kabourakis, Janette L. Vardy

Data analysis and interpretation: Victoria J. Bray, Haryana M. Dhillon, Melanie L. Bell, Mallorie H. Fiero, Desmond Yip, Frances Boyle, Janette L. Vardy

Manuscript writing: All authors

Final approval of manuscript: All authors

\section{REFERENCES}

1. Boykoff N, Moieni M, Subramanian SK: Confronting chemobrain: An in-depth look at survivors' reports of impact on work, social networks, and health care response. J Cancer Surviv 3:223-232, 2009

2. Castellon SA, Ganz PA, Bower JE, et al: Neurocognitive performance in breast cancer survivors exposed to adjuvant chemotherapy and tamoxifen. J Clin Exp Neuropsychol 26:955-969, 2004

3. Jenkins V, Shilling V, Deutsch G, et al: A 3-year prospective study of the effects of adjuvant treatments on cognition in women with early stage breast cancer. Br J Cancer 94:828-834, 2006

4. Ahles TA, Root JC, Ryan EL: Cancer- and cancer treatment-associated cognitive change: An update on the state of the science. J Clin Oncol 30: 3675-3686, 2012

5. Levine $B$, Robertson $I H$, Clare $L$, et al: Rehabilitation of executive functioning: An experimentalclinical validation of goal management training. J Int Neuropsychol Soc 6:299-312, 2000

6. Craik FI, Winocur G, Palmer $\mathrm{H}$, et al: Cognitive rehabilitation in the elderly: Effects on memory. J Int Neuropsychol Soc 13:132-142, 2007

7. Ferguson RJ, Ahles TA, Saykin AJ, et al: Cognitive-behavioral management of chemotherapyrelated cognitive change. Psychooncology 16:772-777, 2007

8. Ferguson RJ, McDonald $B C$, Rocque $M A$, et al: Development of CBT for chemotherapy-related cognitive change: Results of a waitlist control trial. Psychooncology 21:176-186, 2012

9. Kesler S, Hadi Hosseini SM, Heckler C, et al: Cognitive training for improving executive function in chemotherapy-treated breast cancer survivors. Clin Breast Cancer 13:299-306, 2013

10. Mahncke HW, Connor BB, Appelman J, et al: Memory enhancement in healthy older adults Using a brain plasticity-based training program: A randomized, controlled study. Proc Natl Acad Sci USA 103: 12523-12528, 2006

11. Smith GE, Housen $P$, Yaffe $K$, et al: A cognitive training program based on principles of brain plasticity: Results from the Improvement in Memory with Plasticity-based Adaptive Cognitive Training (IMPACT) study. J Am Geriatr Soc 57:594-603, 2009

12. Kim SJ, Stasio C, Spina LM, et al: Enhanced quality of life in individuals with "chemobrain" after using a brain-plasticity-based training program. Psychooncology 17:S96, 2008

13. Jacobs SR, Jacobsen PB, Booth-Jones $M$, et al: Evaluation of the functional assessment of cancer therapy cognitive scale with hematopoietic stem cell transplant patients. J Pain Symptom Manage 33:13-23, 2007

14. BrainHQ.com: Brain training that works. http:// www.brainhq.com/?lead_id=bing-search-text-homeBrand_(US_CAN_UK_AUS_SAF_NZ)\&utm_source = bing\&utm_medium $=$ cpc\&utm_campaign $=$ Brand $\%$ 20(US\%2FCAN\%2FUK\%2FAUS\%2FSAF\%2FNZ\% 2FIN)\&utm_term=brainhq.com\&utm_content=Brain HO

15. Schagen SB, van Dam FS, Muller MJ, et al: Cognitive deficits after postoperative adjuvant chemotherapy for breast carcinoma. Cancer 85:640-650, 1999

16. Wagner $L$, Sweet J, Butt $Z$, et al: Measuring patient self-reported cognitive function: Development of the Functional Assessment of Cancer Therapy - Cognitive Function instrument. J Support Oncol 7:W32-W39, 2009

17. Vardy J, Wong K, Yi QL, et al: Assessing cognitive function in cancer patients. Support Care Cancer 14:1111-1118, 2006

18. Collie A, Maruff P, Makdissi M, et al: CogSport: Reliability and correlation with conventional cognitive tests used in postconcussion medical evaluations. Clin J Sport Med 13:28-32, 2003

19. Goldberg DP, Williams P: A User's Guide to the General Health Questionnaire. Windsor, United Kingdom, NFER-Nelson, 1988

20. Cella DF, Tulsky DS, Gray G, et al: The Functional Assessment of Cancer Therapy scale: Development and validation of the general measure. J Clin Oncol 11: 570-579, 1993

21. Cella D: The Functional Assessment of Cancer Therapy-Anemia (FACT-An) Scale: A new tool for the assessment of outcomes in cancer anemia and fatigue. Semin Hematol 34(3, Suppl 2)13-19, 1997

22. Cohen S, Kamarck T, Mermelstein R: A global measure of perceived stress. J Health Soc Behav 24: 385-396, 1983

23. Fitzmaurice GM, Laird NM, Ware JH: Applied Longitudinal Analysis. Hoboken, NJ, Wiley, 2011

24. Webster K, Cella D, Yost K: The Functional Assessment of Chronic Illness Therapy (FACIT) measurement system: Properties, applications, and interpretation. Health Qual Life Outcomes 1:79-85, 2003

25. Von Ah D, Carpenter JS, Saykin A, et al: Advanced cognitive training for breast cancer survivors: A randomized controlled trial. Breast Cancer Res Treat 135:799-809, 2012

26. King S, Green HJ: Psychological intervention for improving cognitive function in cancer survivors: A literature review and randomized controlled trial. Front Oncol 5:72, 2015

27. Ercoli LM, Petersen L, Hunter AM, et al: Cognitive rehabilitation group intervention for breast cancer survivors: Results of a randomized clinical trial. Psychooncology 24:1360-1367, 2015

28. Cherrier MM, Anderson $K$, David $D$, et al: $A$ randomized trial of cognitive rehabilitation in cancer survivors. Life Sci 93:617-622, 2013

29. Wefel JS, Vardy J, Ahles T, et al: International Cognition and Cancer Task Force recommendations to harmonise studies of cognitive function in patients with cancer. Lancet Oncol 12:703-708, 2011

30. Joly F, Giffard B, Rigal O, et al: Impact of cancer and its treatments on cognitive function: Advances in research from the Paris International Cognition and Cancer Task Force symposium and update since 2012. J Pain Symptom Manage 50: 830-841, 2015

\section{Affiliations}

Victoria J. Bray, Liverpool Hospital; University of Sydney; Haryana M. Dhillon, Melanie L. Bell, and Michael Kabourakis, Centre for Medical Psychology \& Evidence-based Decision-making, School of Psychology, University of Sydney; Frances Boyle and Melanie A. Price, University of Sydney; Frances Boyle, Patricia Ritchie Centre for Cancer Care and Research; Janette L. Vardy, Concord Cancer Centre; Sydney Medical School, University of Sydney, Sydney, New South Wales; Desmond Yip, Australian National University, Canberra, Australian Capital Territory, Australia; and Melanie L. Bell and Mallorie H. Fiero, University of Arizona, Tucson, AZ.

\section{Support}

Supported by the Cancer Council New South Wales, Friends of the Mater Foundation, a Cancer Institute New South Wales Clinical Fellowship (V.J.B.), a Clinical Oncology Society of Australia/Roche Hematology Oncology Targeted Therapies Fellowship (V.J.B.), a Pfizer Cancer Research Grant (V.J.B.), and by the National Breast Cancer Foundation (J.L.V.). 
AUTHORS' DISCLOSURES OF POTENTIAL CONFLICTS OF INTEREST

Evaluation of a Web-Based Cognitive Rehabilitation Program in Cancer Survivors Reporting Cognitive Symptoms After Chemotherapy

The following represents disclosure information provided by authors of this manuscript. All relationships are considered compensated. Relationships are self-held unless noted. I = Immediate Family Member, Inst = My Institution. Relationships may not relate to the subject matter of this manuscript. For more information about ASCO's conflict of interest policy, please refer to www.asco.org/rwc or ascopubs.org/jco/site/ifc.

Victoria J. Bray

Consulting or Advisory Role: Pfizer

Travel, Accommodations, Expenses: Boehringer Ingelheim

Haryana M. Dhillon

Research Funding: Merck Sharp \& Dohme

Melanie L. Bell

No relationship to disclose

Michael Kabourakis

No relationship to disclose

\section{Mallorie H. Fiero}

No relationship to disclose

Desmond Yip

No relationship to disclose

Frances Boyle

No relationship to disclose

Melanie A. Price

No relationship to disclose

Janette L. Vardy

Travel, Accommodations, Expenses: Teva Pharmaceutical Industries 


\section{Acknowledgment}

Presented in part at the 51st Annual Meeting of the American Society of Clinical Oncology, Chicago, IL, May 29-June 2, 2015; the Annual Scientific Meeting of the Medical Oncology Group Australia, Hobart, Tasmania, Australia, August 5-7, 2015; the 42nd Annual Meeting of the Clinical Oncology Society of Australia, Hobart, Tasmania, Australia, November 17-19, 2015; and the International Cognition and Cancer Task Force meeting, Amsterdam, the Netherlands, March 14-16, 2016.

We acknowledge in-kind support from Posit Science for provision of the Insight programs and thank Cogstate for use of their cognitive testing materials. We also thank the Breast Cancer Network Australia and the National Breast Cancer Foundation for their assistance with participant recruitment through their respective Review and Survey and Register 4 groups. Finally, we thank collaborators, study coordinators, and participants from the following hospitals: Concord Repatriation General Hospital, Royal Prince Alfred Hospital, Strathfield Private Hospital, The Mater Hospital, Liverpool Hospital, Campbelltown Hospital, Southern Highlands Cancer Centre, Dubbo Base Hospital, North Coast Cancer Institute, The Canberra Hospital, Flinders Medical Centre, Royal Brisbane Hospital, The Wesley Hospital, Cairns Base Hospital, Border Medical Oncology, Bendigo Oncology and Haematology Services, Ballarat Oncology, and Launceston Hospital.

\section{Appendix}

\section{Missing Data Investigation}

We compared baseline characteristics between participants who were missing and those who were observed after the intervention (T2) for the primary outcome, self-reported cognitive function, measured by the Functional Assessment of Cancer Therapy Cognitive Function (FACT-COG) perceived cognitive impairment (PCI) subscale, and objective neuropsychological function assessed by Cogstate. We used $t$ tests to compare continuous variables, including age, years of education, and time since completion of chemotherapy. We used $\chi^{2}$ tests to compare categorical variables, including married/de facto relationship, smoking history (PCI only), radiotherapy, immune therapy, hormone therapy (yes/no), previous neurologic history, previous use of antidepressants, and current use of antidepressants. We used Fisher's exact test to compare categorical variables with small expected cell frequencies, including sex, English as first language, smoking history (Cogstate only), primary tumor type, and hormone therapy.

Participants who had missing PCI at T2 were younger ( $49 v 54$ years old, $P=.001$ ) and had a higher proportion of previous $(26 \% v 19 \%, P=.01)$ and current $(34 \% v 19 \%, P=.02)$ antidepressant use compared with those who were observed (Table A1). Participants who had missing Cogstate at T2 had fewer years of education (13v14 years, $P=.01)$ and a higher proportion of previous $(56 \% v 35 \%, P=.0008)$ and current $(31 \% v 14 \%, P=.002)$ antidepressant use compared with those who were observed (Table A2).

\section{Sensitivity Analysis for Missing Data: Multiple Imputation and Adjusted Model}

As a sensitivity analysis for missing data, we performed multiple imputation (MI) for the primary outcome, self-reported cognitive function, measured by the FACT-COG PCI subscale. MI reflects uncertainty in predictions of missing data by drawing a set of values, as opposed to a single value, from a predictive distribution for each missing observation. Analysis is performed on each data set, and the results are combined using Rubin's rules (Rubin DB: Multiple imputation for nonresponse in surveys. John Wiley \& Sons, 2004).

Table A3 displays the amount of missing outcome data by treatment arm and follow-up. All patients had complete PCI data at baseline. After the intervention, 23 participants in the control arm (19\%) and 27 in the intervention arm (22\%) had missing PCI data. At 6 months after the intervention, 32 in the control arm (26\%) and 26 in the intervention arm (21\%) had missing PCI data.

Imputation was performed in wide form, with a single data row for each participant, so that correlation within participant was maintained. For the imputation model, we used arm (intervention, control), stratification (five strata on the basis of primary tumor type and use/nonuse of hormonal therapies for patients with breast cancer), and other patient-reported outcomes collected during the trial, including each of the FACT-COG subscales (impact on quality of life, comments from others, perceived cognitive abilities), the Functional Assessment of Cancer Therapy-General total score, Functional Assessment of Cancer Therapy-Fatigue subscale, General Health Questionnaire, and Perceived Stress Scale to help predict values for the missing PCI data. We also considered covariates thought to be associated with missing data and the outcome using logistic regression and mixed models, respectively. The covariates considered were age, antidepressant use (ever or current), smoking history (never, previous, current), tumor stage ( 1 to 3), previous cognitive problems (yes, no), number of chemotherapy cycles (continuous), and time since last chemotherapy (continuous). A participant was considered to have previous cognitive problems if he or she indicated at least one of the following: being held back a year in school, remedial help required at school, a learning disability diagnosis, head injury with loss of 
consciousness, residual sequalae such as headaches or blurred vision, seizures, epilepsy, dementia, cardiac arrest requiring cardiopulmonary resuscitation, coma, stroke, history of other neurologic risk, or history of significant alcohol abuse. Of the covariates, only age, antidepressant use, and tumor stage were found to be associated with the outcome. Tumor stage was associated with missing data but led to convergence issues during the imputation procedure; therefore, age and antidepressant use, together with the aforementioned variables, were used in the imputation models.

We used the MI procedure in SAS statistical software version 9.4 (SAS Institute, Cary, NC). We used the Markov chain Monte Carlo method, which assumes that the data have a multivariate normal distribution and completes 200 burn-in iterations before the first imputation and 100 iterations between imputations. We imputed all missing values and used a single chain to produce 20 imputations. All imputed values were rounded to the nearest whole number and were constrained to each variable's possible range. MIs were performed by treatment arm. Using the completed data set, we analyzed PCI using linear mixed models adjusting for baseline PCI and stratification. After the intervention and at follow-up, the overall difference (and 95\% CIs) between treatment arms and means for each treatment arm and time point were calculated using Rubin's rules as the average of estimates from each of the 20 MI data sets.

We also fitted an adjusted model, using our primary model, as well as several baseline variables including age, antidepressant use, education, months since chemotherapy, previous cognitive problems, baseline stress, and baseline fatigue.

Our analysis using MI yielded results similar to those of the primary analysis (Table A4). When MI was used, a slightly smaller difference between treatment arms was seen after the intervention (difference, $-7.0 ; 95 \% \mathrm{CI},-10.2$ to $-3.9 ; P<.001$ ) and 6 months after the intervention (difference, $-6.4 ; 95 \% \mathrm{CI},-9.8$ to $-3.1 ; P<.001$ ). The adjusted model also gave similar results, although they were slightly stronger. 


\begin{tabular}{|c|c|c|c|}
\hline Characteristic & Observed $(n=192)$, No. $(\%)$ & Missing $(n=50)$, No. $(\%)$ & $P$ \\
\hline \multicolumn{4}{|l|}{ Sex } \\
\hline Female & $182(95)$ & $48(96)$ & \multirow[t]{2}{*}{.73} \\
\hline Male & $10(5)$ & $2(4)$ & \\
\hline Age, years, median (range) & $54(31-74)$ & $49(23-72)$ & .001 \\
\hline \multicolumn{4}{|l|}{ Married/de facto relationship } \\
\hline Yes & $153(80)$ & 39 (78) & \multirow[t]{3}{*}{.91} \\
\hline No & $16(9)$ & $7(14)$ & \\
\hline Unknown & $13(7)$ & $4(8)$ & \\
\hline Education, years, median (range) & $14(3-19)$ & $13(9-19)$ & .43 \\
\hline \multicolumn{4}{|l|}{ English as first language } \\
\hline Yes & $187(97)$ & 47 (94) & \multirow[t]{2}{*}{.37} \\
\hline No & 5 (3) & $3(6)$ & \\
\hline \multicolumn{4}{|l|}{ Smoking history } \\
\hline Never & $110(57)$ & $25(50)$ & \multirow[t]{4}{*}{.46} \\
\hline Previous & 74 (39) & $74(24)$ & \\
\hline Current & 7 (4) & $1(2)$ & \\
\hline Unknown & $1(1)$ & $0(0)$ & \\
\hline \multicolumn{4}{|l|}{ Primary tumor type } \\
\hline Breast & $172(90)$ & $44(88)$ & \multirow[t]{7}{*}{.21} \\
\hline Colorectal & $12(6)$ & $1(2)$ & \\
\hline Gynecologic & $3(2)$ & $2(4)$ & \\
\hline Lymphoma & $2(1)$ & $1(2)$ & \\
\hline Thoracic & $2(1)$ & $1(2)$ & \\
\hline Upper Gl & $1(1)$ & $0(0)$ & \\
\hline Other & $0(0)$ & $1(2)$ & \\
\hline Time since completion of chemotherapy, months, mean (range) & $26(6-60)$ & $30(8-51)$ & .08 \\
\hline \multicolumn{4}{|l|}{ Radiotherapy } \\
\hline Yes & $128(67)$ & $36(72)$ & \multirow[t]{2}{*}{.47} \\
\hline No & 64 (33) & $14(28)$ & \\
\hline \multicolumn{4}{|l|}{ Immune therapy } \\
\hline Yes & $40(21)$ & $14(28)$ & \multirow[t]{2}{*}{.28} \\
\hline No & $152(79)$ & $36(72)$ & \\
\hline \multicolumn{4}{|l|}{ Hormone therapy } \\
\hline No & $56(29)$ & $17(34)$ & \multirow{6}{*}{$\begin{array}{l}.51 \\
.06\end{array}$} \\
\hline Yes & $136(71)$ & $33(66)$ & \\
\hline Tamoxifen & $56(29)$ & $14(28)$ & \\
\hline Letrozole & $24(13)$ & $9(18)$ & \\
\hline Anastrazole & $49(26)$ & $5(10)$ & \\
\hline Other & $7(4)$ & $5(10)$ & \\
\hline \multicolumn{4}{|l|}{ Previous neurologic history* } \\
\hline Yes & $37(41)$ & $30(60)$ & \multirow[t]{2}{*}{.30} \\
\hline No & $114(59)$ & $20(40)$ & \\
\hline Ever used antidepressants & & & \\
\hline Yes & $78(19)$ & $13(26)$ & .01 \\
\hline No & $155(81)$ & $37(74)$ & \\
\hline Current use of antidepressants & & & \\
\hline Yes & $36(19)$ & $17(34)$ & .02 \\
\hline No & $156(81)$ & $33(66)$ & \\
\hline Patient-reported outcomes, mean (SD) & & & \\
\hline Perceived cognitive impairments (FACT-COG PCI) & $39.9(15.1)$ & 41.5 (13.4) & .46 \\
\hline Perceived cognitive abilities (FACT-COG PCA) & $12.3(5.0)$ & $11.8(6.4)$ & .61 \\
\hline Comments from others (FACT-COG comments) & $3.3(3.9)$ & $2.7(2.9)$ & .24 \\
\hline Cognitive quality of life (FACT-COG OOL) & $7.3(4.2)$ & $8.7(4.8)$ & .06 \\
\hline Fatigue (FACT-F) & $33.1(10.8)$ & $28.4(12.1)$ & .02 \\
\hline Quality of life (FACT-G) & $78.0(14.0)$ & $72.4(16.4)$ & .03 \\
\hline Anxiety and depression (GHQ) & $26.5(5.9)$ & $28.2(5.6)$ & .07 \\
\hline Stress (PSS) & $24.4(4.9)$ & $26.4(4.9)$ & .01 \\
\hline $\begin{array}{l}\text { Abbreviations: CRP, cognitive rehabilitation program; FACT-CO } \\
\text { Cancer Therapy-Fatigue; FACT-G, Functional Assessment of C } \\
\text { perceived cognitive impairment; PSS, Perceived Stress Scale; } \\
\text { *Defined as held back a grade in school; required remedial hel } \\
\text { sequelae; history of seizures, dementia, coma, epilepsy, cardia } \\
\text { significant alcohol abuse. }\end{array}$ & $\begin{array}{l}\text { I Assessment of Cancer Therap } \\
\text { py-General; GHQ, General Hea } \\
\text { of life; T2, after intervention. } \\
\text { diagnosed with a learning disab } \\
\text { uiring cardiopulmonary resuscit }\end{array}$ & $\begin{array}{l}\text { Function; FACT-F, Function } \\
\text { nnaire; PCA, perceived cogr } \\
\text { njury with loss of consciousn } \\
\text { e; history of other neurolog }\end{array}$ & $\begin{array}{l}\text { ent of } \\
; \mathrm{PCl} \text {, } \\
\text { sidual } \\
\text { ory of }\end{array}$ \\
\hline
\end{tabular}


Bray et al

Table A2. Baseline Characteristics of Participants With Observed and Missing Cogstate at T2

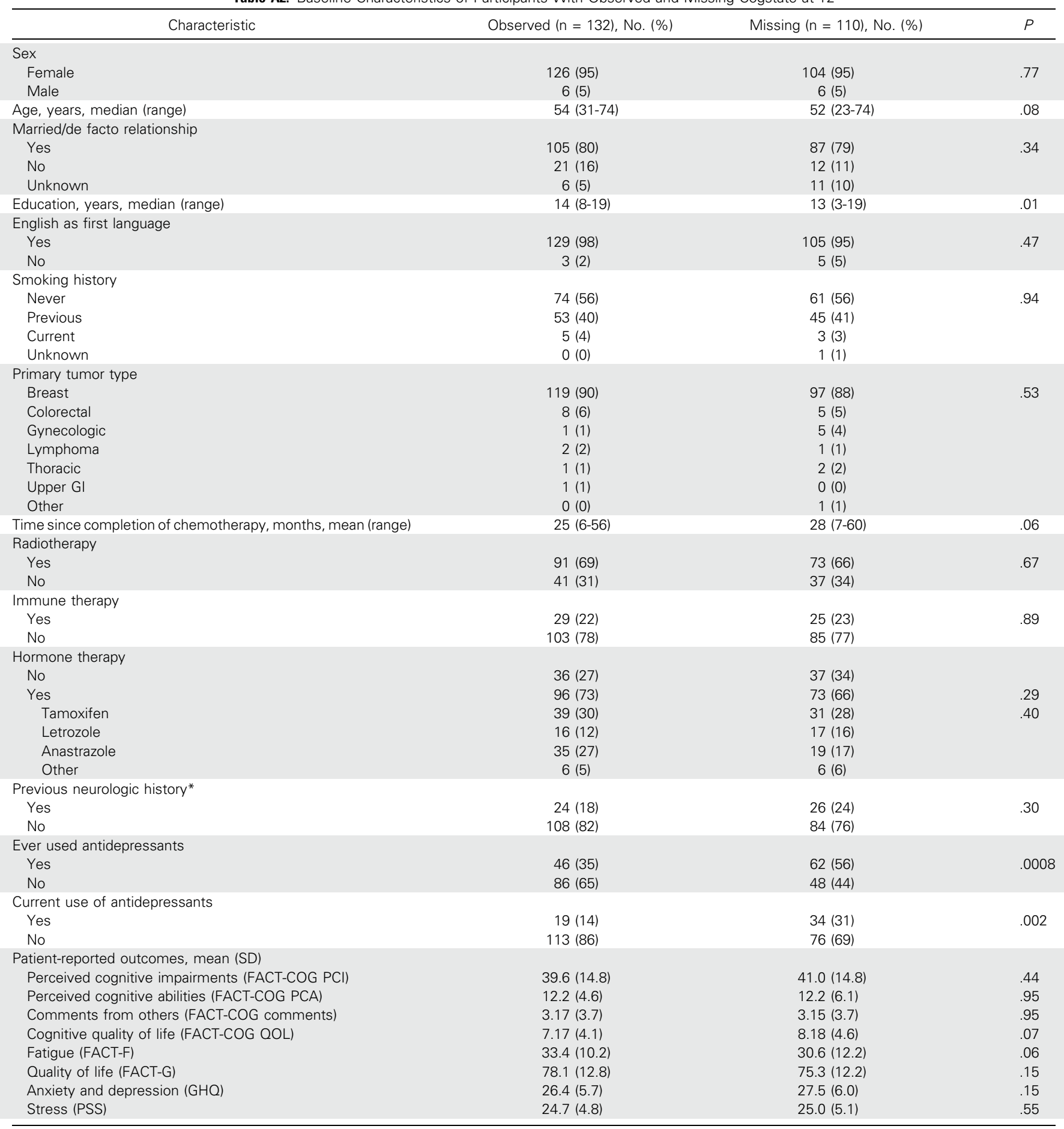

Abbreviations: CRP, cognitive rehabilitation program; FACT-COG, Functional Assessment of Cancer Therapy Cognitive Function; FACT-F, Functional Assessment of Cancer Therapy-Fatigue; FACT-G, Functional Assessment of Cancer Therapy-General; GHQ, General Health Questionnaire; PCS, perceived cognitive abilities; PCI, perceived cognitive impairment; PSS, Perceived Stress Scale; QOL, quality of life; T2, after intervention.

*Defined as held back a grade in school; required remedial help at school; diagnosed with a learning disability; head injury with loss of consciousness with residual sequelae; history of seizures, dementia, coma, epilepsy, cardiac arrest requiring cardiopulmonary resuscitation, stroke; history of other neurologic risk; history of significant alcohol abuse. 
Table A3. Missing Data of Perceived Cognitive Impairments by Treatment Arm and Time

Time of Assessment $\operatorname{CRP}(n=121)$, No. (\%) Control $(n=121)$, No. $(\%)$

\begin{tabular}{ccc}
\hline T1 & $0(0)$ & $0(0)$ \\
T2 & $27(22)$ & $23(19)$ \\
T3 & $26(21)$ & $32(26)$
\end{tabular}

Abbreviations: CRP, cognitive rehabilitation program; T1, baseline; T2, after intervention; T3, 6 months after intervention.

\begin{tabular}{|c|c|c|c|c|}
\hline Analysis/Time of Assessment & CRP & Control & Difference $(95 \% \mathrm{Cl})$ & $P$ \\
\hline \multicolumn{5}{|l|}{ Primary analysis } \\
\hline T1 & 39.6 & 42.9 & - & - \\
\hline T2 & 26.0 & 33.5 & $-7.5(-10.8$ to -4.1$)$ & $<.001$ \\
\hline T3 & 25.8 & 32.3 & $-6.5(-9.9$ to -3.1$)$ & $<.001$ \\
\hline \multicolumn{5}{|l|}{ Multiple imputation } \\
\hline T1 & 40.4 & 41.2 & - & - \\
\hline $\mathrm{T} 2$ & 26.1 & 33.1 & $-7.0(-10.2$ to -3.9$)$ & $<.001$ \\
\hline T3 & 26.5 & 32.0 & $-6.4(-9.8$ to -3.1$)$ & $<.001$ \\
\hline \multicolumn{5}{|l|}{ Adjusted model } \\
\hline $\mathrm{T} 1$ & 40.5 & 41.4 & - & - \\
\hline $\mathrm{T} 2$ & 25.5 & 33.7 & $-8.2(-11.7$ to -4.7$)$ & $<.001$ \\
\hline T3 & 25.3 & 32.5 & $-7.2(-10.7$ to -3.7$)$ & $<.001$ \\
\hline
\end{tabular}

Abbreviations: CRP, cognitive rehabilitation program; T1, baseline; T2, after intervention; T3, 6 months after intervention. 